\title{
Prognostic impact of IKZF1 deletion in adults with common B-cell acute lymphoblastic leukemia
}

Qiu-Mei Yao', Kai-Yan Liu', Robert Peter Gale², Bin Jiang ${ }^{1}$, Yan-Rong Liu', Qian Jiang ${ }^{1}$, Hao Jiang ${ }^{1}$, Xiao-Hui Zhang ${ }^{1}$, Mei-Jie Zhang ${ }^{3}$, Shan-Shan Chen ${ }^{1}$, Xiao-Jun Huang ${ }^{1,4}$, Lan-Ping X (1*t $^{1{ }^{*}}$ and Guo-Rui Ruan ${ }^{1{ }^{*+}}$

\begin{abstract}
Background: Interrogate the impact of IKZF1 deletion on therapy-outcomes of adults with common B-cell acute lymphoblastic leukemia.

Methods: One hundred sixty-five consecutive adults with common B-cell ALL were tested for IKZF1 deletion and for BCR/ABL. Deletions in IKZF1 were detected using multiplex RQ-PCR, multiplex fluorescent PCR, sequence analysis and multiplex ligation-dependent probe amplification (MLPA). BCR/ABL was detected using RQ-PCR. All subjects received chemotherapy and some also received an allotransplant and tyrosine kinase-inhibitors. Multivariate analyses were done to identify associations between IKZF1 deletion and other variables on non-relapse mortality (NRM), cumulative incidence of relapse (CIR), leukemia-free survival (LFS) and survival.

Results: Amongst subjects achieving complete remission those with IKZF1 deletion had similar 5-year non-relapse mortality (NRM) (11\% [2-20\%] vs. $16 \%[4-28 \%] ; P=0.736)$, a higher 5-year cumulative incidence of relapse (CIR) (55\% [35-76\%] vs. $25 \%$ [12-38\%]; $P=0.004)$, and worse 5-year leukemia-free survival (LFS) (33\% [16-52\%] vs. $59 \%$ [42-73\%]; $P=0.012$ ) and survival (48 \% [33-62\%] vs. $75 \%$ [57-86\%]; $P=0.002$ ). In multivariate analyses IKZF1 deletion was associated with an increased relapse (relative risk [RR] $=2.7,[1.4-5.2] ; P=0.002$ ), a higher risk of treatment-failure (inverse of LFS; $R R=2.1,[1.2-3.6] ; P=0.007$ ) and a higher risk of death $(R R=2.8,[1.5-5.5] ; P=0.002)$. The adverse impact of IKZF1 deletion on outcomes was stronger in subjects without vs. with BCR-ABL1 and in subjects receiving chemotherapy-only vs. an allotransplant.

Conclusions: IKZFI deletion was independently-associated with a higher relapse risk and worse LFS and survival in adults with common B-cell ALL after adjusting for other prognostic variables and differences in therapies. These data suggest IKZF1 deletion may be a useful prognostic variable in adults with common B-cell ALL, especially in persons without BCR-ABL1 and those receiving chemotherapy-only. Transplants appear to overcome the adverse impact of IKZF1 deletion on therapy-outcomes but confirmation in a randomized study is needed. The trial was registered in 2007 with the Beijing Municipal Government (Beijing Municipal Health Bureau Registration N: 2007-1007).
\end{abstract}

Keywords: IKZFI, Acute lymphoblastic leukemia, BCR-ABL1, Chemotherapy, Allotransplant

\footnotetext{
*Correspondence: Ipxu_0415@sina.com; ruanguorui@pkuph.edu.cn

${ }^{\dagger}$ Equal contributors

${ }^{1}$ Beijing Key Laboratory of Hematopoietic Stem Cell Transplantation, Peking

University People's Hospital and Institute of Hematology, 11 Xi-Zhi-Men

South Street, 100044 Beijing, China

Full list of author information is available at the end of the article
} 


\section{Background}

About $85 \%$ of cases of adult acute lymphoblastic leukemia (ALL) develop from B-cells. Identification of biological determinants of treatment-outcomes is incomplete. Consensus prognostic variables include age, gender, WBC level at diagnosis, cytogenetic abnormalities, $B C R-A B L 1$, central nervous system (CNS) leukemia, time to initial response and detection of measureable residual disease (MRD) after induction and consolidation therapies [1-5]. However, analyses of receiver-operator characteristic (ROC) curves indicate only about one-half of the variability in subject-level outcomes is explained by known prognostic variables [6].

Genome-wide analyses indicate $I K Z F 1$ mutations are common in ALL and are associated with $B C R-A B L 1[7,8]$. $I K Z F 1$ encodes the Ikaros transcription factor, a member of a family of zinc-finger nuclear proteins required for normal lymphoid development [9]. Intra-genic deletions in $I K Z F 1$ generate aberrant isoforms $[8,10]$. The role of $I K Z F 1$ deletions is extensively-evaluated in children with ALL and is the most common genetic marker associated with a poor prognosis [11-15]. IKZF1 mutations are also common in adults with B-cell ALL and are also associated with poor prognosis in persons with concurrent $B C R$ $A B L 1$ [16-18]. Few data are available on the role of IKZF1 deletions in adults with common B-cell ALL. In preliminary analyses we found frequent IKZF1 deletions in this population. Based on these data we interrogated associations between $I K Z F 1$ deletion and prognosis in a series of adults with common B-cell ALL.

\section{Methods}

\section{Subjects}

Between April, 2007 and December, 2012165 newlydiagnosed subjects $\geq 14$ years old with common B-cell ALL were seen at Peking University People's Hospital, Peking University Institute of Hematology (Beijing, China). 3 subjects with severe co-morbidities were ineligible for induction chemotherapy ( 2 had an $I K Z K 1$ deletion) and excluded from further analyses. The 162 eligible subjects received remission-induction chemotherapy. Those achieving complete remission received 2 cycles of consolidation chemotherapy after which they could continue receiving chemotherapy or receive an allotransplant based on donor availability, finances and physician and subject choice. Physicians and subjects were blinded to results of IKZF1testing. Subjects were followed until death, loss to follow-up or December, 2014. Written informed consent was obtained from adult subjects and from parents of minors in accordance with the Declaration of Helsinki. The study was approved by the Ethics Committee of Peking University People's Hospital. Subjects receiving post-remission chemotherapy were included in a Chinese Clinical Trial registered in 2007 with the Beijing Municipal
Government (Beijing Municipal Health Bureau Registration N: 2007-1007).

\section{Diagnosis and response criteria}

Diagnosis of ALL was based on $\geq 20 \%$ bone marrow lymphoblasts. Common-B-cell type was defined as cases with CD10, CD19, CD22 and CD79a expression and no surface immunoglobulin by flow cytometry [19]. Complete remission was defined bone marrow lymphoblasts $<5 \%$, neutrophils $>1.0 \times 10 \mathrm{E}+9 / \mathrm{L}$, platelets $>100 \times 10 \mathrm{E}+9 / \mathrm{L}$, no extra-medullary disease and no loss of these features for $>4$ weeks. Relapse was defined as leukemia recurrence at any site in persons in complete remission.

\section{Cytogenetic and molecular analyses}

Cytogenetic analyses were performed by using standard G-banding [20]. $B C R-A B L 1$ transcripts, $M L L$ rearrangement and $E 2 A / P B X 1$ transcripts were detected with quantitative real-time polymerase chain reaction (RQPCR) [21, 22]. IKZF1 deletions were detected using multiplex RQ-PCR, multiplex fluorescent PCR, sequence analysis [23] and multiplex ligation-dependent probe amplification (MLPA) (MRC-Holland, Amsterdam, Netherlands) performed using SALSA MLPA kit P202-B1 IKZF1. Focal IKZF1 gene deletions (deletions of exons 4-7, 4-8, 2-7, 28 and other focal deletions identified by MLPA analysis) were defined as $I K Z F 1$ deletion in the present study.

\section{Chemotherapy}

One hundred six subjects without BCR/ABL received induction chemotherapy with 1-2 cycles of CODPL (cyclophosphamide, vincristine, daunorubicin, prednisone and L-asparaginase). 56 subjects with $B C R-A B L 1$ received induction chemotherapy with the same regimen without L-asparaginase and 48 also received imatinib, $400 \mathrm{mg} / \mathrm{d}$, beginning at diagnosis. 5 were later switched to dasatinib, 1 to nilotinib and 1 to ponatinib because of resistant mutations. Data regarding IKZF1 deletion were not used to determine induction chemotherapy. Subjects achieving complete remission (CR) received 2 cycles consolidation chemotherapy with hyper-CVAD (cyclophosphamide, vincristine, doxorubincin, dexamethasone, cytarabine and methotrexate). All subjects received CNS prophylaxis with intrathecal methotrexate, cytarabine and dexamethasone for $\geq 8$ doses during induction and consolidation therapy. Details of these regimens are reported [24]. Subjects remaining in CR continued receiving chemotherapy until the completed 6 more cycles, received an allotransplant or relapsed. Post-consolidation therapy was with 6-mercaptopurine, $50 \mathrm{mg} / \mathrm{mE} 2 / \mathrm{d}$ PO and methotrexate, $20 \mathrm{mg} / \mathrm{mE} 2 / \mathrm{d} \mathrm{PO}$, each once weekly. 28 subjects with $B C R-A B L 1$ receiving an allotransplant also received posttransplant tyrosine kinase-inhibitor (TKI) therapy. 17 subjects with $B C R-A B L 1$ receiving post-consolidation 
chemotherapy but no allotransplant also received postconsolidation TKI therapy. Decisions to give or not to give TKI therapy post-consolidation or posttransplant were based on finances and subject and/or physician preference and without knowing results of IKZF1 deletion-testing.

\section{Allotransplants}

Ninety-two subjects (64\%) received an allotransplant, 24 (26\%) from an HLA-identical sibling and 68 (74\%) from a HLA-haplotype-mismatched related donor (1 HLA-antigen mismatch $[N=4], 2$ HLA-antigen mismatches $[N=20]$ and 3 HLA-antigen mismatches $[N=$ 44]). Conditioning was with busulfan and cyclophosphamide. Anti-thymocyte globulin was given pretransplant when the donor was a HLA-haplotype-mismatched relative. Donors received recombinant human G-CSF after which bone marrow and blood cells were collected and infused into the recipient. Graft-vs.-host disease prophylaxis was with cyclosporine, mycophenolatemofetil and short-term methotrexate. Haematological relapses were treated with donor mononuclear cells infusions. Some subjects received a $2^{\text {nd }}$ transplant from the same or a different donor. Details are reported $[25,26]$.

\section{Endpoints and statistical analyses}

Haematologic response was analyzed weekly for the first 3 months and monthly thereafter. Cytogenetic and molecular responses were analyzed every 3 months for the first 6 months and every 6-12 months thereafter. Leukemia-free survival (LFS) was calculated from the date of $1^{\text {st }}$ complete remission to the date of first relapse or death in complete remission. Survival was calculated from $1^{\text {st }}$ complete remission to the date of death from any cause. Observations were censored at the date of last contact or December, 2014 when no events were observed. In survival analyses subjects receiving an allotransplant who relapsed were censored at the time they received a donor blood cell infusion or a second allotransplant. Last follow-up was December, 2014. Relapse was defined as $1^{\text {st }}$ hematologic relapse regardless of site. Independence of categorized parameters was calculated using Chi-square test (or Fisher Exact test). Distribution of continuous variables was calculated using Wilcoxon two sample tests. Survival functions were estimated by the Kaplan-Meier method and compared by the log-rank test. Cumulative incidences were estimated for NRM and relapse to accommodate competing risks. NRM was a competing risk for relapse and death from any cause was a competing risk for relapse. A Cox proportional hazard regression model was used to determine associations between IKZF1 deletion and other variables with relapse, NRM, treatment-failure and death. The variables to be considered in the multivariate models were: $I K Z F 1$ deletion $(\mathrm{Y} / \mathrm{N}), B C R-A B L 1(\mathrm{Y} / \mathrm{N})$, gender, age ( $\geq$ vs. $<35$ years),
WBC ( $\geq$ vs. $<30 \times 10 \mathrm{E}+9 / \mathrm{L}$ ), hypo-diploid karyotype $(\mathrm{Y} / \mathrm{N})$, complex karyotype and treatment (chemotherapy vs. allotransplant). CNS, $M L L$ rearrangement or $E 2 A-P B X 1$ transcripts and TKI were not considered in the multivariate analysis because of either too few subjects in a category or missing data. Assumption of proportional hazards for each factor in the Cox model was tested using time-dependent covariates. A stepwise model selection approach was used to identify all significant risk factors. Each step of model building contained the main effect for IKZF1 deletion. Factors which are significant at a $P=0.05$ level were kept in the final model. Potential interactions between main effect and all significant risk factors were tested. Analyses were performed by SPSS software version 19.0 (Chicago, IL, USA), Graphpad Prism 5.01 (San Diego, California, USA) and R version 3.1.2. Difference with $P<0.05$ was considered significant.

\section{Results \\ Subjects}

Sixty-eight IKZF1 local deletions were detected of subjects achieving complete remission including 32 (47\%) subjects with type $\Delta 4-7,17$ (25\%) with type $\Delta 2-7,3$ with type $\Delta 4-8,10(15 \%)$ with type $\Delta 2-8$ and 6 with other deletions. 6 other deletions identified by MLPA analysis were undetectable by multiplex PCR. Proportions of dominant negative isoforms $(\Delta 4-7$ deletion) and haplotype-insufficiency (other local deletion types) were comparable. The proportion of $\Delta 4-7$ deletion in subjects with $B C R-A B L 1$ was higher than in subjects without $B C R-A B L 1$ ( $20 / 31$ vs. $12 / 37 ; P=0.008)$. Baseline subjectand disease-related variables of subjects achieving $C R$ are shown in Table 1 . Subjects with IKZF1 deletion were older (34 years [15-70] vs. 22 [14-59]; $P=0.045$ ), had a significantly higher WBC (14 [2-435] vs. 9 [0.5-168]; $P=0.008)$ and were more likely to have BCR-ABL1 $(75 \%$ vs. $25 \% ; P<0.01)$ and $\mathrm{t}(9 ; 22)(72 \%$ vs. $28 \% ; P<0.05)$ than subjects without IKZF1 deletion.

\section{Outcomes and IKZF1 deletion}

Median follow-up was 22 months (range, 2-91 months). $146(90 \%)$ subjects achieved complete remission. 2 subjects in the IKZF1 cohort relapsing during consolidation chemotherapy and before they could be assigned to further chemotherapy-only or an allotransplant were excluded from further analyses. 5-year NRM in the 144 subjects was $14 \%$ (95\% confidence interval [CI], 6-22\%), 5-year CIR was $38 \%$ (27-49\%), 5-year LFS, $48 \%(36-59 \%)$ and 5-year survival, $63 \%(51-72 \%)$. In subjects with $I K Z F 1$ deletion 5-year NRM was $11 \%$ (2-20 \%) vs. $16 \%(4-28 \% ; P=0.736$, Fig. 1a) for subjects without IKZFI deletion. In subjects with IKZF1 deletion 5-year CIR was $55 \%(35-76 \%)$ vs. $25 \%(12-38 \%$; 
Table 1 Subject- and disease-related variables

\begin{tabular}{|c|c|c|c|c|}
\hline Variable & All subjects & IKZF1-deleted & IKZF1 wild type & $P$-value \\
\hline$N$ & 146 & 68 (47\%) & 78 (53 \%) & \\
\hline \multicolumn{5}{|l|}{ Age, y } \\
\hline Median (range) & $27(14-70)$ & $34(15-70)$ & $22(14-59)$ & 0.045 \\
\hline \multicolumn{5}{|l|}{ Gender } \\
\hline Male & 72 & $33(46 \%)$ & 39 (54 \%) & 0.859 \\
\hline \multicolumn{5}{|l|}{ WBC $(\times 10 E+9 / L)$} \\
\hline Median (range) & $10(0.5-435)$ & $14(2-435)$ & $9(0.5-168)$ & 0.008 \\
\hline \multicolumn{5}{|l|}{ Hemoglobin (g/L) } \\
\hline Median (range) & $90(23-157)$ & $92(35-148)$ & $89(23-157)$ & 0.300 \\
\hline \multicolumn{5}{|l|}{ Platelets (×10E + 9/L) } \\
\hline Median (range) & $57(0.2-313)$ & $58(0.2-311)$ & $52(2-313)$ & 0.600 \\
\hline \multicolumn{5}{|c|}{ Bone marrow blasts, $\%$} \\
\hline Median (range) & 87 (28-99) & $86(28-98)$ & $88(28-99)$ & 0.300 \\
\hline$B C R-A B L 1$ & 52 & $39(75 \%)^{\mathrm{a}}$ & $13(25 \%)^{b}$ & 0.000 \\
\hline \multicolumn{5}{|l|}{ Cytogenetics } \\
\hline Normal & 47 & 19 (40 \%) & $28(60 \%)$ & 0.305 \\
\hline Abnormal & 81 & 38 (47 \%) & 43 (53 \%) & \\
\hline$t(9 ; 22)$ & 43 & 31 (72 \%) & $12(28 \%)$ & 0.000 \\
\hline$t(X ; 2)(p 22 ; p 21)$ & 1 & 1 & 0 & \\
\hline $\operatorname{del}(9)(p 13)$ & 1 & 1 & 0 & \\
\hline $\operatorname{inv}(9)$ & 2 & 1 & 1 & \\
\hline $47,+x$ & 1 & 1 & 0 & \\
\hline Complex & 33 & 3 & 30 & \\
\hline Unknown & 18 & $11(61 \%)$ & 7 (39\%) & \\
\hline
\end{tabular}

a8 subjects with $B C R$-ABL1 had no $\mathrm{t}(9 ; 22)$ including 5 with insufficient metaphases, 2 with an abnormal karyotype but not $\mathrm{t}(9 ; 22)$ and 1 with a normal karyotype ${ }^{b} 1$ subject with $B C R-A B L 1$ had a normal karyotype

$P=0.004$; Fig. 1b) for subjects without IKZF1 deletion. In subjects with $I K Z F 1$ deletion 5-year LFS was $33 \%(16-52 \%)$ vs. $59 \%(42-73 \% ; P=0.012$; Fig. 1c) in subjects without IKZF1 deletion. In subjects with IKZF1 deletion 5-year survival was $48 \%$ (33-62 \%) vs. $75 \%(57-86 \% ; P=0.002$; Fig. $1 \mathrm{~d})$ in subjects without IKZF1 deletion. There were 26 deaths in $I K Z F 1$ deletion cohort, 20 from leukemia and 6 from other causes. There were 15 deaths in the no IKZF1 deletion cohort, 5 from leukemia and 10 from other causes. Prognoses were similar in subjects with a dominant negative IKZF1 isoform compared to haplotype-insufficient subjects: 4-year NRM $14 \%(1-27 \%)$ vs. $8 \%(0-19 \%$; $P=0.32)$; 4-year CIR $50 \%(29-70 \%)$ vs. $44 \%$ (23-64\%; $P=0.54)$; 4-year LFS $36 \%(18-55 \%)$ vs. $49 \%$ (28-67\%; $P=0.20)$ and 4-year survival $45 \%(23-65 \%)$ vs. $51 \%$ (30-69\%; $P=0.53)$.

Because IKZF1 deletion and BCR-ABL1 were confounded ( $r=0.407 ; P<0.001)$, we analyzed the impact of $I K Z F 1$ deletion in subjects with and without $B C R-A B L 1$. Amongst the 94 subjects without $B C R-A B L 1$ the 65 subjects with $I K Z F 1$ deletion had a significantly higher 4-year CIR than the 29 subjects without IKZF1 deletion (51 \% [30-72\%] vs. $17 \%$ [7-28\%]; $P=0.002)$. These subjects also had worse LFS (33\% [15-52\%] vs. $72 \%$ [58-82\%]; $P=0.001$ ) and worse survival (46\% [24$66 \%$ ] vs.84 \% [71-91\%]; $P=0.000)$. There was no significant difference in their NRM (17 \% [1-32 \%] vs. $11 \%$ [2-19\%]; $P=0.525)$. In contrast, there were no significant differences in NRM, CIR, LFS or survival in subjects with $B C R-A B L 1$ with and without IKZF1 deletion.

\section{Interaction between IKZF1 deletion and post-remission therapy}

Fifty-two subjects achieving remission received chemotherapy-only. 3-year NRM was $15 \%$ (3-26\%), 3-year CIR, 53 \% (36-70 \%), 3-year LFS, 32 \% (17-49\%) and 3-year survival, $44 \%$ (23-64 \%). Subjects with IKZF1 deletion $(N=28)$ had similar 3-year NRM (14 \% [0-29\%] vs. $15 \%[0-33 \%] ; P=0.958), 3$-year CIR (73\% [41$100 \%$ vs. $39 \%$ [16-61\%]; $P=0.099)$, worse 3-year LFS (14\% [1-41\%] vs. $46 \%$ [23-66 \%]; $P=0.084)$ and 

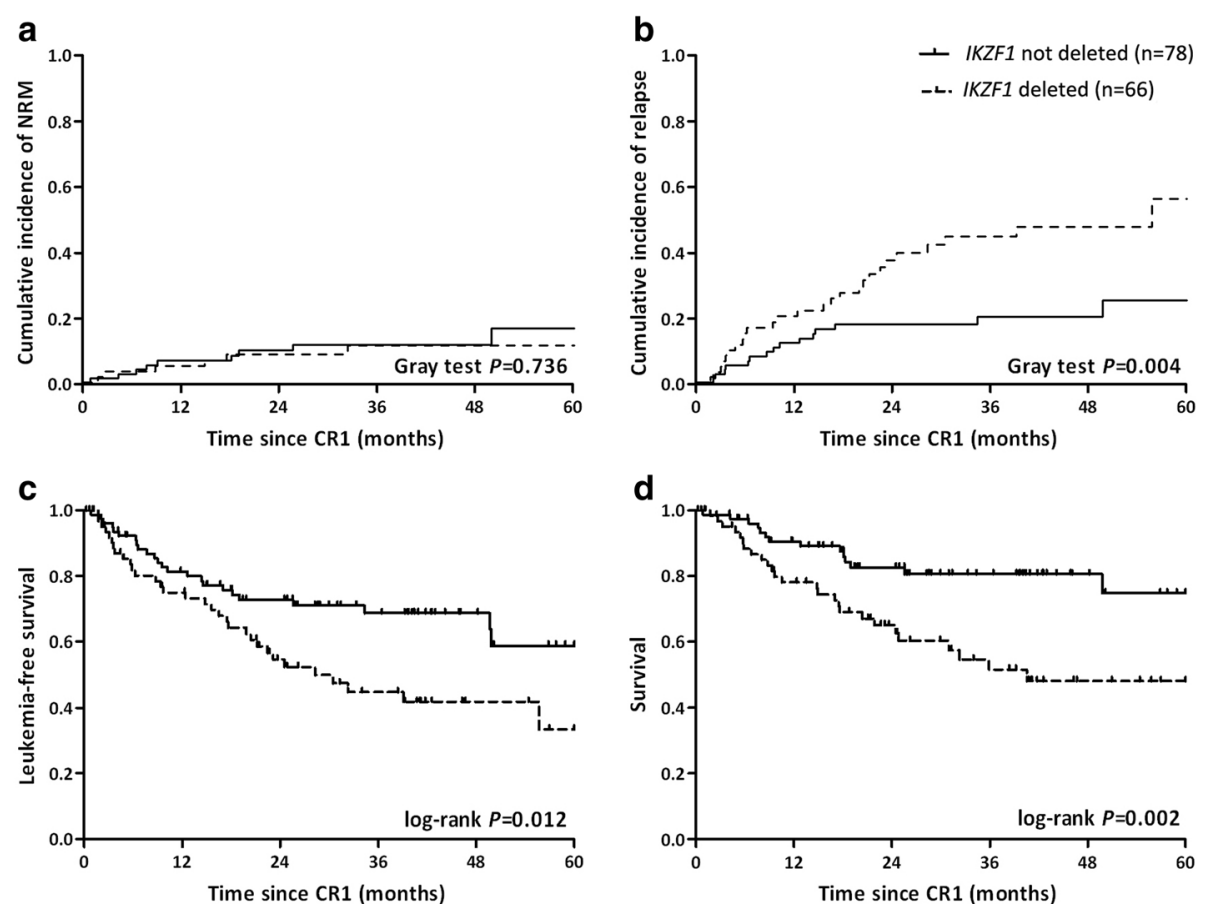

Fig. 1 a Cumulative incidence of non-relapse mortality (NRM); b cumulative incidence of relapse (CIR); c leukemia-free survival (LFS); and $\mathbf{d}$ survival of subjects with and without IKZFI deletion since first complete remission

worse 3-year survival (15\% [1-44 \%] vs.78 \% [48-92\%]; $P=0.001$; Fig. 2a-d) compared with subjects without IKZF1 deletion.

Ninety-two (64\%) subjects achieving remission received an allotransplant. 5-year NRM was $14 \%$ (4-24 \%), 5-year CIR, $31 \%$ (17-45\%), 5-year LFS, $56 \%$ (40-70\%) and 5-year survival, $71 \%$ (56-81 \%). Subjects with IKZF1 deletion $(N=38)$ had a significantly higher 5 year CIR (47\% [21-73\%] vs. $20 \%$ [3-36\%]; $P=0.024)$ but similar 5 years NRM (9 \% [0-19 \%] vs. $17 \%$ [1-33 \%]; $P=0.567)$, 5-year LFS (44 \% [21-66 \%] vs. $64 \%$ [40-80\%]; $P=0.119)$ and 5-year survival (64 \% [44-79 \%] vs.75 \% [53-88\%]; $P=$ 0.332; Fig. 2e-h).

\section{Multivariate analyses}

$I K Z F 1$ deletion was independently associated with an increased relapse risk $(R R=2.7[1.4-5.2] ; P=0.002)$, a greater risk of treatment-failure $(R R=2.1[1.2-3.6] ; P=$ $0.007)$ and a greater risk of death $(R R=2.8$ [1.5-5.5]; $P=0.002)$. An adverse impact of $I K Z F 1$ on survival was observed in subjects receiving chemotherapy-only $(R R=$ 6.5 [2.1-19.7]; $P=0.001$ ) but not those receiving an allotransplant $(R R=1.5[0.7-3.6] ; P=0.336)$. Other variables associated with relapse were chemotherapy-only $(R R=6.0$ [3.1-11.6]; $P<0.0001)$ and male gender $(R R=$ $2.0[1.1-3.9] ; P=0.030)$. Chemotherapy-only was also associated with greater risk of treatment-failure $(R R=4.4$ [2.6-7.6]; $P<0.0001)$ and death $(R R=4.1$ [2.1-7.8]; $P<$ $0.0001)$. Age $\geq 35$ years was the only variable associated with NRM $(R R=3.0$ [1.1-8.7]; $P=0.039)$. Results of multivariate analyses are shown in Table 2.

\section{Discussion}

IKZF1 deletion was independently-associated with higher risks of relapse, treatment-failure and death in adults with common B-cell ALL compared with similar subjects without $I K Z F 1$ deletion. Although $I K Z F 1$ deletion was confounded with $B C R-A B L 1$, the adverse impact persisted after adjusting for $B C R-A B L 1$ and parallels similar findings in children with B-cell ALL $[12,15]$. The adverse impact of $I K Z F 1$ deletion operated mainly in subjects without $B C R-A B L 1$. In subjects receiving an allotransplant the adverse impact of IKZF1 deletion was less than in those receiving chemotherapy-only suggesting a benefit for using transplants in subjects with IKZF1 deletion.

Our analysis was complex because of confounding between $I K Z F 1$ deletion, $B C R-A B L 1$ and post-remission therapy (chemotherapy-only vs. an allotransplant). We used multivariate analyses to resolve this confounding. We found IKZF1deletion, chemotherapy-only, gender and age were significantly associated with outcomes whereas other variables including WBC, cytogenetics and $B C R-A B L 1$ 

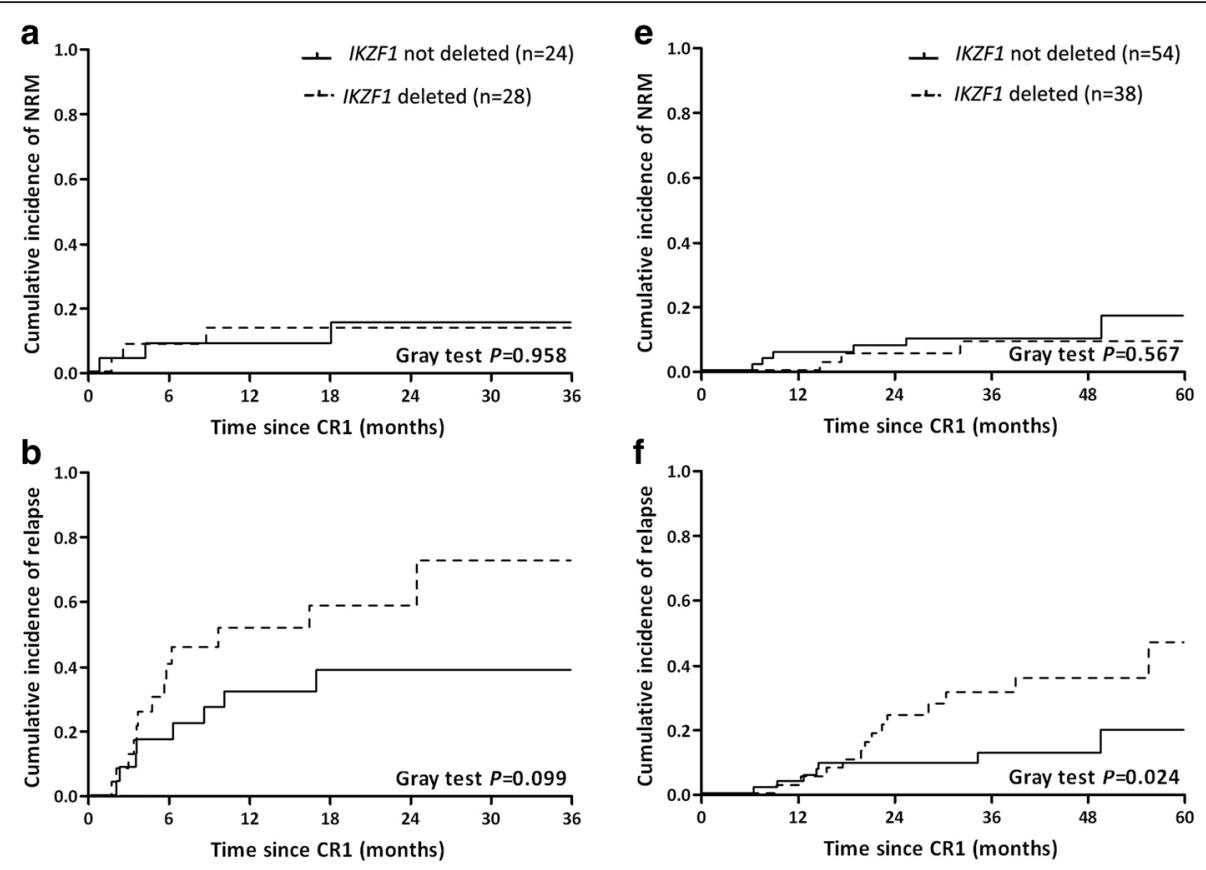

C

\section{g}
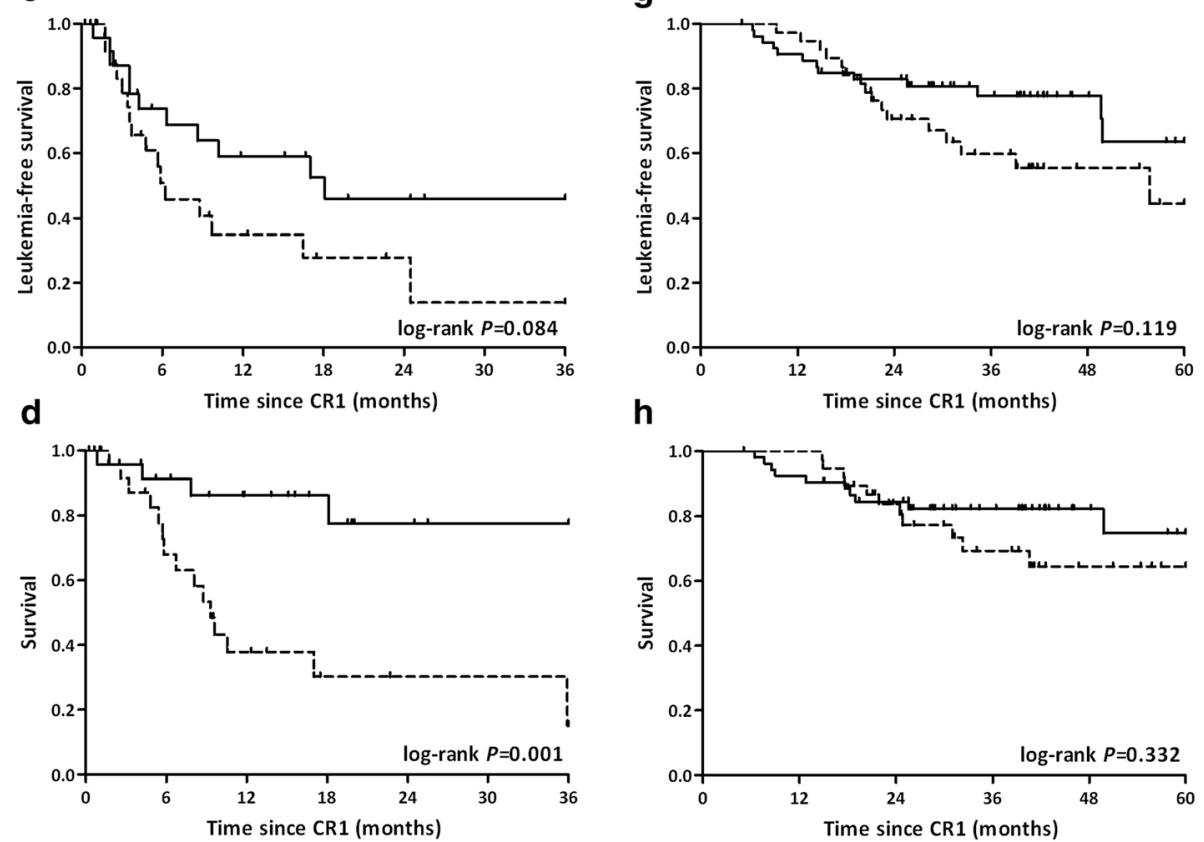

Fig. 2 NRM, CIR, LFS and survival of subjects with and without IKZFI deletion receiving chemotherapy-only (a-d) or an allotransplant (e-h) since $1^{\text {st }}$ complete remission. Abbreviations: NRM, non-relapse mortality; CIR, cumulative incidence of relapse; LFS, leukemia-free survival; CR1, $1^{\text {st }}$ complete remission

were not. This is surprising, especially the lack of a significant association between $B C R-A B L 1$ with outcomes. There are several possible explanations. One is insufficient statistical power; there were relatively few subjects with adverse cytogenetics, hypo-diploidly or a complex karyotype. However, low power cannot explain the lack of a significant association between $B C R-A B L 1$ and outcomes. As indicated, IKZF1 deletion and BCR-ABL1 were confounded. Subjects with $B C R-A B L 1$ received different initial therapy than subjects without $B C R$ $A B L 1$ including TKIs in $90 \%$ and an allotransplant in $58 \%$. If these interventions were highly-effective they could mitigate the adverse impact of $B C R-A B L 1$. This notion is supported by the observation the adverse 
Table 2 Multivariate analyses of NRM, relapse, treatment-failure and death

\begin{tabular}{|c|c|c|c|}
\hline Outcome & $N$ & $\mathrm{RR}(95 \% \mathrm{Cl})$ & $P$ \\
\hline \multicolumn{4}{|l|}{ NRM } \\
\hline IKZF1 deleted vs. not deleted & $66 / 78$ & $0.8(0.3-2.2)$ & 0.630 \\
\hline Age: $\geq$ vs. $<35$ y & $55 / 89$ & $3.0(1.1-8.7)$ & 0.040 \\
\hline \multicolumn{4}{|l|}{ Relapse } \\
\hline IKZF1 deleted vs. not deleted & $66 / 78$ & $2.7(1.4-5.2)$ & 0.002 \\
\hline Chemotherapy vs. transplant & $52 / 92$ & $6.0(3.1-11.6)$ & 0.000 \\
\hline Male vs. female & $73 / 71$ & $2.0(1.1-3.9)$ & 0.030 \\
\hline \multicolumn{4}{|l|}{ Treatment-failure } \\
\hline IKZF1 deleted vs. not deleted & $66 / 78$ & $2.1(1.2-3.6)$ & 0.007 \\
\hline Chemotherapy vs. transplant & $52 / 92$ & $4.4(2.6-7.6)$ & 0.000 \\
\hline \multicolumn{4}{|l|}{ Death } \\
\hline IKZF1 deleted vs. not deleted & $66 / 78$ & $2.8(1.5-5.6)$ & 0.002 \\
\hline Chemotherapy vs. transplant & $52 / 92$ & $4.1(2.1-7.8)$ & 0.000 \\
\hline \multicolumn{4}{|l|}{ Chemotherapy } \\
\hline IKZF1 deleted vs. not deleted & $28 / 24$ & $6.5(2.1-19.7)$ & 0.001 \\
\hline \multicolumn{4}{|l|}{ Transplant } \\
\hline IKZF1 deleted vs. not deleted & $38 / 54$ & $1.5(0.7-3.6)$ & 0.336 \\
\hline
\end{tabular}

predictive impact of IKZF1 deletion was detected in subjects without but not in those with $B C R-A B L 1$ and in subjects receiving chemotherapy-only but not in those receiving an allotransplant.

Our conclusions contrast with data from a study of 83 subjects with $B C R-A B L 1$ which reported $I K Z F 1$ deletions were associated with more relapses and worse LFS [16]. However, there are several important differences in subjects (we studied only subjects with common B-cell ALL) and post-remission therapy (few of their subjects received an allotransplant). Our conclusion is similar to reports in children with $B C R-A B L 1$ in whom $I K Z F 1$ deletion is associated with worse outcomes and with results of a recent meta-analysis indicating IKZF1 deletion is independentlyassociated with worse outcomes in children and adults with ALL $[27,28]$.

There are several limitations to our study. One, as discussed, is confounding between $I K Z F 1$ deletion, $B C R$ $A B L 1$ and post-remission therapy. Confounding is difficult to satisfactorily sort out in multivariate analyses but our data suggest that IKZF1 deletion was independentlyassociated with higher risks of relapse, treatment-failure and death in subjects without $B C R-A B L 1$. Second, subjects with $B C R-A B L 1$ received different induction, consolidation and post-remission therapies than those without $B C R-A B L 1$. However, therapy-assignment was made without knowing IKZF1 deletion data and we tried to account for this complexity in multivariate analyses. Another limitation is we did not include several potentially important variables (CNS leukemia, $M L L$ rearrangement, $E 2 A-P B X 1$ transcripts and TKI therapy) in the multivariate analyses because of too few subjects or missing data. The sum of these potential limitations means our conclusions require confirmation in prospective, randomized studies.

\section{Conclusion}

In adults with common B-cell ALL achieving complete remission IKZF1 mutation was independently-associated with a higher CIR and worse survival than subjects without IKZF1 mutation. These differences were greater in subjects receiving post-remission chemotherapy than in subjects receiving an allotransplant. We suggest a randomized trial to confirm our observation.

\section{Abbreviations}

MLPA: multiplex ligation-dependent probe amplification; NRM: non-relapse mortality; CIR: cumulative incidence of relapse; LFS: leukemia-free survival; ALL: acute lymphoblastic leukemia; CNS: central nervous system;

ROC: receiver-operator characteristic; MRD: measureable residual disease; TKI: tyrosine kinase-inhibitor; CR: complete remission; RQ-PCR: quantitative real-time polymerase chain reaction.

\section{Competing interests}

The authors declare that they have no competing interests.

\section{Authors' contributions}

LPX, GRR and RPG designed the project and prepared the typescript. QMY performed the PCR experiments and all statistical analyses. KYL, BJ, YRL, QJ, $\mathrm{HJ}, \mathrm{XHZ}$, SSC and XJH fulfilled ethical authorizations, collected and stored clinical data. MJZ helped with the multivariate analyses. All authors approved the typescript

\section{Acknowledgements}

Profs. Charles Mullighan (St. Jude Children Hospital) and Christine Harrison (Royal Victoria Infirmary) kindly reviewed the typescript. Supported by grants from the National Basic Research Program of China (grant 2013CB733701), the Key Program of National Natural Science Foundation of China (grant 81230013), the National Natural Science Foundation of China (grant 81170484), the Beijing Municipal Natural Science Foundation (grant 7122199) and the Specialized Research Fund for the Doctoral Program of Higher Education of China (grant 20130001110079). RPG acknowledges support from the NIHR Biomedical Research Centre funding scheme.

\section{Author details}

${ }^{1}$ Beijing Key Laboratory of Hematopoietic Stem Cell Transplantation, Peking University People's Hospital and Institute of Hematology, 11 Xi-Zhi-Men South Street, 100044 Beijing, China. ${ }^{2}$ Haematology Research Center, Division of Experimental Medicine, Department of Medicine, Imperial College London, London, UK. ${ }^{3}$ Division of Biostatistics, Medical College of Wisconsin, Milwaukee, USA. ${ }^{4}$ Peking-Tsinghua Center for Life Sciences, Beijing, China.

Received: 2 July 2015 Accepted: 1 April 2016

Published online: 11 April 2016

\section{References}

1. Gokbuget N, Hoelzer D. Treatment of adult acute lymphoblastic leukemia. Hematology Am Soc Hematol Educ Program. 2006:133-41

2. Chiaretti S, Vitale A, Cazzaniga G, Orlando SM, Silvestri D, Fazi P, et al. Clinico-biological features of 5202 patients with acute lymphoblastic leukemia enrolled in the Italian AIEOP and GIMEMA protocols and stratified in age cohorts. Haematologica. 2013;98:1702-10.

3. Moorman AV, Harrison CJ, Buck GA, Richards SM, Secker-Walker LM, Martineau $M$, et al. Karyotype is an independent prognostic factor in adult acute lymphoblastic leukemia(ALL): analysis of cytogenetic data from patients treated on the Medical Research Council (MRC) UKALLXII/Eastern Cooperative Oncology Group (ECOG) 2993 trial. Blood. 2007;109:3189-97. 
4. Burke PW, Douer D. Acute lymphoblastic leukemia in adolescents and young adults. Acta Haematol. 2014;132:264-73.

5. Lazarus HM, Luger $\mathrm{S}$. Which patients with adult acute lymphoblastic leukemia should undergo a hematopoietic stem cell transplantation? Case-Based Discussion. Hematology Am Soc Hematol Educ Program. 2007:444-52

6. Walter RB, Othus M, Burnett AK, Löwenberg B, Kantarjian HM, Ossenkoppele GJ, et al. Resistance prediction in AML: analysis of 4601 patients from MRC/NCRI, HOVON/SAKK, SWOG and MD Anderson Cancer Center. Leukemia. 2015;29:312-20.

7. Mullighan CG, Goorha S, Radtke I, Miller CB, Coustan-Smith E, Dalton JD, et al. Genome-wide analysis of genetic alterations in acute lymphoblastic leukaemia. Nature. 2007;446:758-64.

8. Mullighan CG, Miller CB, Radtke I, Phillips LA, Dalton J, Ma J, et al. BCR-ABL1 lymphoblastic leukaemia is characterized by the deletion of Ikaros. Nature. 2008;453:110-4.

9. Dovat S. Ikaros in hematopoiesis and leukemia. World J Biol Chem. 2011;2:105-7.

10. lacobucci I, Storlazzi CT, Cilloni D, Lonetti A, Ottaviani E, Soverini S, et al. Identification and molecular characterization of recurrent genomic deletions on 7p12 in the IKZF1 gene in a large cohort of BCR-ABL1-positive acute lymphoblastic leukemia patients: on behalf of Gruppo Italiano Malattie Ematologiche dell'Adulto Acute Leukemia Working Party (GIMEMA AL WP). Blood. 2009;1 14:2159-67.

11. Yamashita Y, Shimada A, Yamada T, Yamaji K, Hori T, Tsurusawa M, et al. IKZF1 and CRLF2 gene alterations correlate with poor prognosis in Japanese BCR-ABL1-negative high-risk B-cell precursor acute lymphoblastic leukemia. Pediatr Blood Cancer. 2013;60:1587-92.

12. Kuiper RP, Waanders E, van der Velden VH, van Reijmersdal SV, Venkatachalam R, Scheijen B, et al. IKZF1 deletions predict relapse in uniformly treated pediatric precursor B-ALL. Leukemia. 2010;24:1258-64.

13. Mullighan CG, Su X, Zhang J, Radtke I, Phillips LA, Miller CB, et al. Deletion of IKZF1 and prognosis in acute lymphoblastic leukemia. N Engl J Med. 2009:360:470-80.

14. Van der Veer A, Waanders E, Pieters R, Willemse ME, Van Reijmersdal SV, Russell $L$, et al. Independent prognostic value of BCRABL1-like signature and IKZF1 deletion, but not high CRLF2 expression, in children with B-cell precursor ALL. Blood. 2013;122:2622-9.

15. Palmi C, Valsecchi MG, Longinotti G, Silvestri D, Carrino V, Conter V, et al. What is the relevance of Ikaros gene deletions as a prognostic marker in pediatric Philadelphia-negative B-cell precursor acute lymphoblastic leukemia? Haematologica. 2013;98:1226-31.

16. Martinelli G, lacobucci I, Storlazzi CT, Vignetti M, Paoloni F, Cilloni D, et al. IKZF1 (Ikaros) deletions in BCR-ABL1-positive acute lymphoblastic leukemia are associated with short disease-free survival and high rate of cumulative incidence of relapse: A GIMEMA AL WP Report. J Clin Oncol. 2009;27:5202-7.

17. Tokunaga K, Yamaguchi S, Iwanaga E, Nanri T, Shimomura T, Suzushima H, et al. High frequency of IKZFI genetic alterations in adult patients with B-cell acute lymphoblastic leukemia. Eur J Haematol. 2013;91:201-8.

18. Beldjord K, Chevret S, Asnafi V, Huguet F, Boulland ML, Leguay T, et al. Oncogenetics and minimal residual disease are independent outcome predictors in adult patients with acute lymphoblastic leukemia. Blood. 2014;123:3739-49.

19. Bassan R, Gatta G, Tondini C, Willemze R. Adult acute lymphoblastic leukaemia. Crit Rev Oncol Hematol. 2004;50:223-61.

20. Zhang $Y$, He Q, Huang XJ, Jiang H, Yang SM, Lu J, et al. Cytogenetic study on eosinophilia. Zhongguo Shi Yan Xue Ye Xue Za Zhi. 2007;15:454-7.

21. Qin YZ, Liu YR, Zhu HH, Li JL, Ruan GR, Zhang Y, et al. Different kinetic patterns of BCR-ABL transcript levels in imatinib-treated chronic myeloid leukemia patients after achieving complete cytogenetic response. Internatl Lab Hematol. 2008;30:317-23.

22. Beillard E, Pallisgaard N, van der Velden VH, Bi W, Dee R, van der Schoot E, et al. Evaluation of candidate control genes for diagnosis and residual disease detection in leukemic patients using 'real-time' quantitative reverse-transcriptase polymerase chain reaction (RQ-PCR) - a Europe against cancer program. Leukemia. 2003;17:2474-86.

23. Caye A, Beldjord K, Mass-Malo K, Drunat S, Soulier J, Gandemer V, et al. Breakpoint-specific multiplex PCR allows the detection of IKZFI intragenic deletions and minimal residual disease monitoring in B-cell precursor acute lymphoblastic leukemia. Haematologica. 2013;98:597-601.

24. Yan CH, Jiang Q, Wang J, Xu LP, Liu DH, Jiang H, et al. Superior Survival of Unmanipulated haploidentical hematopoietic stem cell transplantation compared with chemotherapy alone used as post-remission therapy in adults with standard-risk acute lymphoblastic leukemia in first complete remission. Bone Marrow Transplant. 2014;20:1314-21.

25. Lu DP, Dong L, Wu T, Huang XJ, Zhang MJ, Han W, et al. Conditioning including anti thymocyte globulin followed by unmanipulated HLA-mismatched/haploidentical blood and marrow transplantation can achieve comparable outcomes with HLA-identical sibling transplantation. Blood. 2006;107:3065-73.

26. Huang XJ, Liu DH, Liu KY, Xu LP, Chen H, Han W, et al. Treatment of acute leukemia with unmanipulated HLA-mismatched/haploidentical blood and bone marrow transplantation. Biol Blood Marrow Transplant. 2009;15:257-65.

27. Van der Veer A, Zaliova M, Mottadelli F, De Lorenzo P, Te Kronnie G, Harrison CJ, et al. IKZF1 status as a prognostic feature in BCR-ABL1-positive childhood ALL. Blood. 2014;123:1691-8.

28. Jia M, Wang ZJ, Li JY, Yang SL, Zhao HZ, Cheng YP, et al. The impact of IKZF1 deletion on the prognosis of acute lymphoblastic leukemia: an updated meta-analysis. Cancer Biomarkers. 2014;14:493-503.

\section{Submit your next manuscript to BioMed Central and we will help you at every step:}

- We accept pre-submission inquiries

- Our selector tool helps you to find the most relevant journal

- We provide round the clock customer support

- Convenient online submission

- Thorough peer review

- Inclusion in PubMed and all major indexing services

- Maximum visibility for your research

Submit your manuscript at www.biomedcentral.com/submit
C Biomed Central 